\title{
The Accuracy of Computed Tomography in Blunt Abdominal Trauma in unusual times
}

\author{
Muslim Kandel Kadeem ${ }^{1}$, Mohanad Abdulretha ${ }^{1}$ \\ ${ }^{1}$ Assist. Prof., Department of General Surgery, College of Medicine, University of Thi-Qar, Iraq
}

\begin{abstract}
Background: A potential research for the evaluation of CT scan accuracy in blunt abdominal trauma was carried out by comparing operative and CT scan observation. About 60 patients undertook the blunt abdominal trauma in three years. CT scan was performed on these stabilized patients for its further comparison with operative results. Moreover, all the documented data comprised of scan results, sex, age and type of injuries.

Aim of Study: The objective of our current research was to discover the accuracy of CT scan results for blunt abdominal trauma which was evaluated by surgeons in duty compare with operative findings.

Results: From 60 patients, 40 pts (66.6\%) underwent surgery. 20 pts (33.3\%) were conservatively managed. 5 pts were died. Hemoperitoneum was perceived in fifty five patients. The patients having little hemoperitoneum on CT scan along with reasonable clinical observations were conventionally managed whereas those patients having huge hemoperitoneum needed surgical investigation. Out of all the patients involved in study, 13 had splenic, 3 pancreatic, 15 hepatic, 4 vascular and 5 renal injuries, however, 12 showed retroperitoneum hematoma.
\end{abstract}

Conclusion: In comparison between clinical monitoring, CT in the evaluation of blunt abdominal trauma with accuracy $86-100 \%$ (over all accuracy 94\%).

Keywords: Computed Tomography; Blunt Abdominal Trauma.

\section{Introduction}

The abdominal trauma is categorized in penetrating and blunt kinds. The penetrating abdominal trauma (PAT) is frequently diagnosed on the basis of clinical symptoms. Whereas, the blunt trauma get missed or postponed due to unclear clinical signs. ${ }^{[1]}$

Thus, it can create problems for individuals of all ages. The recognition of severe intra-abdominal pathology is sometimes perplexing as it is difficult to acknowledge various injuries in initial evaluation and treatment ${ }^{[2]}$

Diagnosis: The most significant concern in evaluating the blunt abdominal trauma is the estimation of hemodynamic stability ${ }^{[3]}$. Moreover, a quick estimation for hemodynamically unstable patients can be achieved with FAST (Focused assessment with sonography trauma) and diagnostic eitoneal lavage (DPL) for hemoperitoneum evaluation. The inconvincible physical examination results leads to the abdominal radiographic studies of stable patients ${ }^{[4,5]}$.

FAST: The FAST examination done in 4 windows of ultrasound: (perihepatic, pelvic, pericardiac, perisplenic) along with the supine patients to detect free fluid or bleeding in any of this 4 windows $(2,3,4,5)$

Computed Tomography: The outcomes of CT scans are essential for solid organ injuries' detection such as liver, kidneys, spleen, as well as for retroperitoneum involving colorectum, pancreas, bladder, diaphragm, and small bowel ${ }^{[6]}$. Likewise CT scan can further expose other related injuries for instance, thoracic cavity injuries, vertebral and pelvic fractures as well.

Scans illustrate brilliant imaging for genitourinary system, pancreas and duodenum by revealing blood quantity in abdomen \& precisely each individual organ. 
But it has some limitations such as marginal sensitivity for hollow viscous, pancreatic and diaphragmatic injuries' diagnosis. These are also considered as costly, time taking and need oral or intravenous contrast which may lead to the severe reactions ${ }^{[7,8]}$
Thus, the CT scans in contrast to the FAST and DPL investigation have capacity for the haemorrhage source determination. Besides this, various retroperitoneal injuries get ignored with DPL \& FAST evaluation [9].

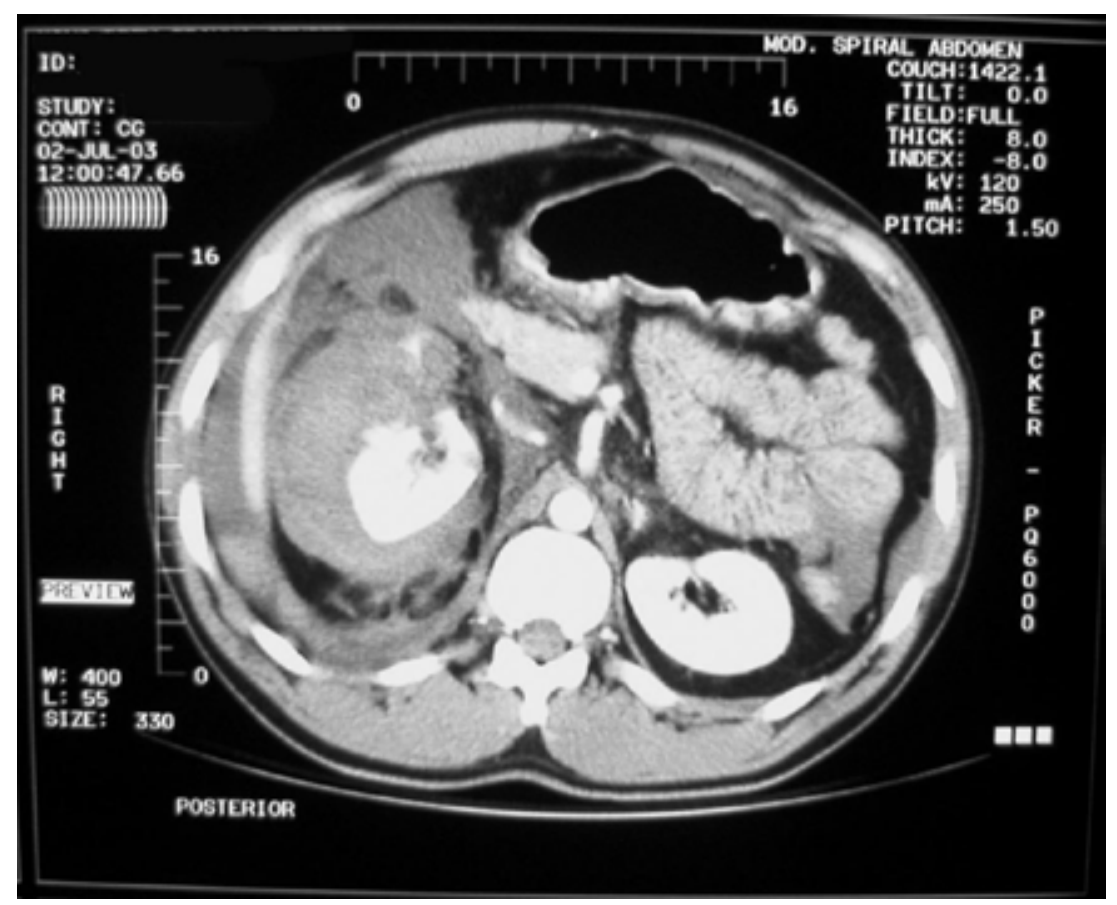

Figure 1. Blunt abdominal trauma. Right kidney injury with blood in perirenal space. Injury resulted from high-speed motor vehicle collision.

\section{Method}

This prospective study, done in Al Hussain Teaching Hospital in Nassyrriah \IRAQ during 3 years (from January 2012 to December 2014), used abdomen CT scan to assess abdominal blunt trauma to most stable patients arrived emergency room in holidays and daily after $10 \mathrm{pm}$ till morning by surgeon in duty and technician (because unavailable radiologist at hospital at this time)

About sixty stabilized blunt abdominal trauma patients went through this examination. The patients included 36 males $(60 \%)$ and 24 females $(40 \%)$. The age range was $7-68$ years.

Clinical examination and diagnostic peritoneal tapping was done for most of them. The patients who weren't admitted or the ones who got discharged after short examination were devoid of any more investigation were omitted from our research.
We are usually looking for any hemoperitonium and solid organ injuries. Hence, the CT of hemoperitoneum was categorised as defined by Federle and Jeffrey et al. ${ }^{[10]}$

- $\quad$ Fluid in one space $=$ Small $(100-200 \mathrm{cc})$

- $\quad$ Fluid in tow spaces or more $=$ Moderate $(250-500 \mathrm{cc})$

- Fluid in all spaces or pelvic fluid anteriorlsuperior tourinary bladder $=$ Large $(>500 \mathrm{cc})$

The Organ Injury Scale (OIS system) was used to grade the solid organ injuries. from simple organic contusion grade I to avascularisation of one organ grade $\mathrm{V}$., then these CT observations were related to the operative discoveries of forty patients who underwent explorative laprotomy .

\section{Results}

Most of patients involved in this study whom 
suffering from abdominal trauma are male, the Male :female ratio is $1.5: 1$ look table 2 they extend from difrrent age groups ranging from 7 years to 64 years.

Table 1. Explain the Gender ratio, mode of injury, operative and conservative management, and organs injuries.

\begin{tabular}{|c|c|c|}
\hline Gender & No of patients & $\%$ \\
\hline Male & 36 & $60 \%$ \\
\hline Female & 24 & $40 \%$ \\
\hline \multicolumn{3}{|l|}{ Mode of injury } \\
\hline Cause of abdominal trauma & No of patients & $\%$ \\
\hline RTA & 48 & $80 \%$ \\
\hline fall from height & 4 & $6,7 \%$ \\
\hline Fighting & 3 & $5 \%$ \\
\hline Animal trauma & 2 & $3.3 \%$ \\
\hline Others & 3 & $5 \%$ \\
\hline \multicolumn{3}{|c|}{ Operative and conservative management } \\
\hline Management & No of patients & Deaths \\
\hline Underwent surgery & 40 patients, $(66.6 \%)$ & 4 \\
\hline Conservatively managed & $20(33.3 \%)$ patients & 1 \\
\hline \multicolumn{3}{|l|}{ Organs injuries } \\
\hline Organs injuries & No of patients & $\%$ \\
\hline Liver & 15 & $37.5 \%$ \\
\hline Spleen & 13 & $32.5 \%$ \\
\hline Kidney & 5 & $12.5 \%$ \\
\hline Bowel \& mesentry & 5 & $12.5 \%$ \\
\hline Pancrease & 3 & $7.5 \%$ \\
\hline Vascular injuries & 4 & $10 \%$ \\
\hline Urinary Bladder & 4 & $10 \%$ \\
\hline Stomach & 2 & $5 \%$ \\
\hline Diaphragmatic rupture & 1 & $2.5 \%$ \\
\hline Retroperitoniumheamatoma & 12 & $30 \%$ \\
\hline
\end{tabular}

The commonest mode of injury in blunt abdominal trauma, is RTA (road traffic accident) in 48 from 60 patients $(80 \%)$. look table1.

Most of patients underwent surgery about 40 from 60 patients, $(66.6 \%)$, while 20 patients $(33.3 \%)$ were conventionally managed without any difficulties. Five deaths occurred and two of them were because of the complications after operations while other two deceased patients were accompanied with sever extra abdominal injuries within first 24 hours . there was one patient not operated because severely injured head and chest, and autopsy reveal no significant intra abdominal injuries look table 1.

Another group with normal CT scan no collection no solid organ injury with stable general condition they discharge home this group not involve in this study

All the 9 patients with large Hemoperitoneum required surgical exploration., while All 10 patients with small fluid on CT scan started with conservative management in spite of one severely injury in head and chest, 3 of them (30\%) deteriorated with in 24 hours of follow up

The moderate fluid group indicated that thirteen (13) were conservatively managed who were stable general condition and the other 28 patients underwent surgical exploration that were either unstable haemodynamically and/or had deterioration of their conditions like unexplained abdominal rigidity, therefore, the explorative laparotomy's rate in moderate hemoperitoneum patients was observed to be as $68 \%$ on CT scan as presented in table (2).

Table 2 (Hemoperitoneum was detected on CT)

\begin{tabular}{|l|c|c|c|c|}
\hline & Total & Conservative & Op & Op \total \\
\hline Small & 10 & 7 & 3 out of 10 & $30 \%$ \\
\hline Moderate & 41 & 13 & 28 out of 41 & $68 \%$ \\
\hline Large & 9 & 0 & 9 & $100 \%$ \\
\hline
\end{tabular}

Most of patient who operated 25 patients from 40 was explored $62.5 \%$ have multiorgans injuries, and 12 patents $30 \%$ have single organ injured, another 3 patents only free fluid no solid organ injured. anyhow the commonest organ involved was the liver 15 out of 40 patients $37.5 \%$ and spleen 13 out of 40 patients $32.5 \%$ look table 1 .

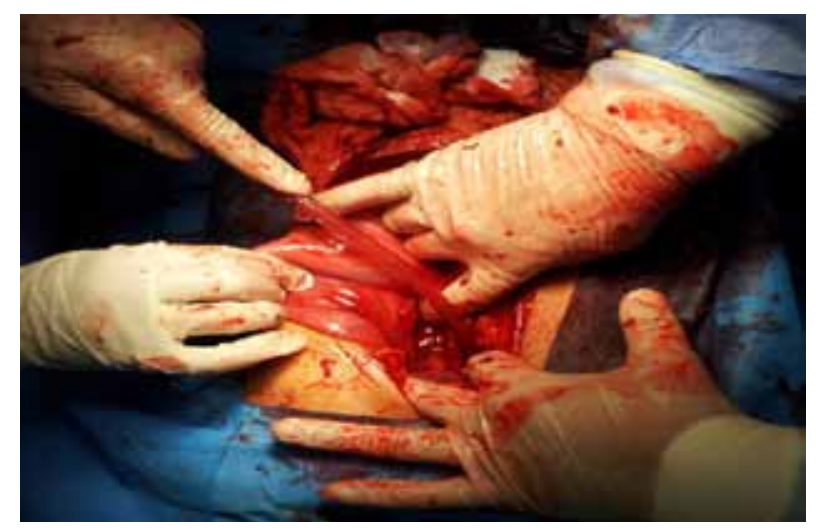

Figure 2. (23 years old man injured by RTA low speed vehicle injury of stomach) 


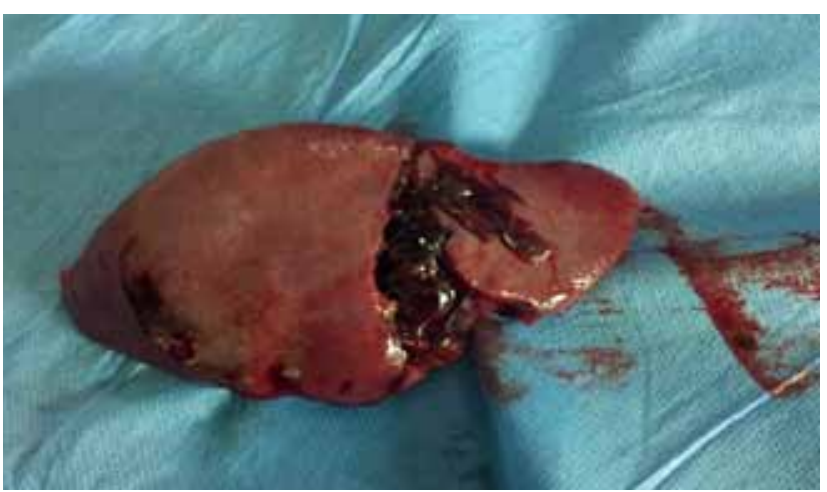

About grading of solid organ injury is its difficult to assess by CT scan by non provisional person and without contrast, but during surgery we found different grading among patient, look table 1 .

About accuracy of fluid collection the accuracy is $100 \%$ all cases we positive in CT scan were positive in surgery, but some cases in CT looked mild collection while in surgery looked moderate or severe, but no false positive or negative

Figure 3. (18 years old woman with splenic injury grade III after fall from height)

Table 3. Show (Grade of solid organ, solid organ and other injuries CT scan findings vs operative findings, fluid collection CT scan findings vs operative findings

\begin{tabular}{|c|c|c|c|c|c|}
\hline Grade solid organ & Liver & Splenic & Kidney & Pancreatic & Total \\
\hline$!$ & 7 & 7 & 1 & 0 & 15 \\
\hline II & 2 & 1 & 2 & 2 & 7 \\
\hline III & 3 & 3 & 2 & 1 & 9 \\
\hline IV & 3 & 1 & 0 & 0 & 4 \\
\hline $\mathrm{V}$ & 0 & 1 & 0 & 0 & 1 \\
\hline Total & 15 & 13 & 5 & 3 & 36 \\
\hline \multicolumn{6}{|c|}{ Solid organ and other injuries CT scan findings vs operative findings } \\
\hline Findings & CT finding & Operative finding & False - ve & False + ve & Accuracy \\
\hline Liver injuries & 13 & 15 & 2 & 0 & $86 \%$ \\
\hline Spleen injuries & 11 & 13 & 2 & 0 & $84 \%$ \\
\hline Kidney injuries & 5 & 5 & 0 & 0 & $100 \%$ \\
\hline Pancreas injuries & 3 & 3 & 1 & 1 & $67 \%$ \\
\hline Diaphragmatic rupture & 1 & 1 & 0 & 0 & $100 \%$ \\
\hline Retroperitonium haematoma & 12 & 12 & 0 & 0 & $100 \%$ \\
\hline \multicolumn{6}{|c|}{ Fluid collection CT scan findings vs operative findings } \\
\hline Findings & CT finding & Operative finding & False - ve & False + ve & Accuracy \\
\hline Mild fluid collection & 3 & 3 & 0 & 0 & $100 \%$ \\
\hline Moderate fluid collection & 28 & 28 & 0 & 0 & $100 \%$ \\
\hline Large fluid collection & 9 & 9 & 0 & 0 & $100 \%$ \\
\hline
\end{tabular}

\section{Discussion}

The primary inspection of abdomen can distinguish the major intra-abdominal haemorrhage symptoms. Whereas, the secondary investigation is important to spot a continuous bleeding or haemorrhage subsequent to the normal blood pressure restoration ${ }^{[11]}$.
The blunt abdominal trauma patients lack the substantial physical symptoms of organ participation. In case of instability of patients having numerous injuries, there is an ambiguity about abdomen being a shock source, thus a FAST investigation might be helpful. In case of stabilized patient and easy approach to CT scan, 
abdomen and head scan should be performed. The stable patients having several injuries including occult organ injuries which are not threat of life then CT estimation is essential. ${ }^{[12]}$ in this study we start with clinical assessment of all patients had abdominal blunt trauma who arrived emergency room, the stable patients sent to CT scan assessment ., if negative or minimal collection they treated conservatively and most of them go home so not involved in this study, but patents whom admit hospital for follow up 60 patients 40 from 60 patients, $(66.6 \%)$ underwent surgery about, while 20 patients (33.3\%) continue on conservative managed without any complications.

Most of patients involved in this study whom suffering from abdominal trauma are male, the Male :female ratio is $1.5: 1$ look table 2 they extend from different age groups ranging from 7 years to 64 years

The severe wound caused because of blunt force trauma are reliant on transferred kinetic energy amount and tissue whom energy is transmitted. Thus the kinetic energy is related to the object movement which is equivalent to the one half of the object mass while multiplying with the square of object velocity such as $1 / 2 \mathrm{mv}^{2}$. Therefore, generally, the lighter objects moving with a high speed are more dangerous as compared to the heavy object roving at lower speed. ${ }^{[1-4]}$ However, unluckily 5 patients expired and out of them two deaths were caused due to postoperative complications. But the other two deceased patients were associated with sever extra abdominal injuries within first 24 hours . there was one patient not operated because severely injured head and chest, and autopsy reveal no significant intra abdominal injuries so all deaths due to severity of trauma look table 1 .

Haemodynamically, the CT accurateness in stabilized blunt trauma patients is well recognised. Moreover, among the patients of emergency CT, the specificity of about $98.7 \%$ while sensitivity of about $97 \%$ $\& 92 \%$ have been testified ${ }^{[13,14]}$ which is near the range of this study table 9 accuracy for solid organs between $84-100 \%$ median range may be because reading by surgeon in duty himself who is not provisional in radiology, and also not use contrast at this time of day but any haw if compare its nearby, Various researchers endorse being admitted and observed subsequent to a negative $\mathrm{CT}^{[15,16]}$ to repeat $\mathrm{CT}$ scan with or without contrast and reassessed by radiologist to exclude farther injuries as CT is particularly insufficient to diagnose the mesenteric wounds while it might skip some hollow visceral wounds. Thus, for the hollow visceral or mesenteric prone patients, the most suitable test is DPL [17]. And negative results of CT scan for these patients do not consistently eliminate the intra-abdominal wounds. So we depend on hemoperitoneum or fluid collection we found the accuracy of CT scan was $100 \%$, the overall nine patients having great hemoperitoneum need surgical examination. Whereas, the group of moderate fluid have conservatively managed thirteen patients who were stable general condition and the other 28 patients underwent surgical exploration. More than $68 \%$ patients of moderate fluid were investigated. Taylor et al. presented a report of an experience of $50 \%{ }^{[18]}$ we think this relatively higher percentage because exclude most of stable patient with negative CT and stable those discharge on their responsibility and not involved in this study, and of course less experience of surgeon in CT scan assessment without contrast at night or holidays.

CT was executed in forty four haemodynamically stabilized patients of blunt trauma succeeding DPL by Kane. The scan of sixteen patients exposed an essential retroperitoneal or intra-abdominal wounds which DPL could not identify. Finally, the results of scans helped $58 \%$ patients in modifying the original plan of treatment. ${ }^{[19]}$

About grading of solid organ injury in this study we cannot assess by CT scan because lack of experience, but during surgery show a lot of patients have multiorgan injuries 25 from 40 patient was explored $62.5 \%$, and 12 patents $30 \%$ have single organ injured, another 3 patents only free fluid no solid organ injured, the injured organs in different grades II, III, IV or V injuries they required surgery. While the rest of the injuries were managed conservatively, and according to follow up clinical finding if deteriorated go to surgery

The high grade injury of solid organs upsurge the surgical management. While the low grade wounds were insufficient for management protocol prediction because some time hypo or hyper assessment of CT scan by surgeon

\section{Conclusion}

We conclude that the CT scan is accurate test for diagnosis of intra-abdominal hemoperitoneum or fluid collections, and solid organ injury after blunt trauma and has all the attributes to make it an initial investigation of choice in haemodynamically stable patients even 
done by surgeon in duty (but it's better to be done with contrast and assessed by provisional radiologist) Thus, the negative laparotomy's rate is decreased by abstaining from unnecessary surgical interference, also reduce missed injury by early discover of internal bleeding or organ injury in cases of conservative management.

Financial Disclosure: There is no financial disclosure.

Conflict of Interest: None to declare.

Ethical Clearance: All experimental protocols were approved under the College of Medicine and all experiments were carried out in accordance with approved guidelines.

\section{References}

1. BMJ. Anson JO, Yule SR, Loudon MA. “2008; 336 (7650): 938-42. doi:10.1136/ bmj.39534.686192.80. PMC 2335258. PMID 18436949 .

2. B J Trauma. (rasel KJ, Olson CJ, Stafford RE, Johnson TJ). Incidence and significance of free fluid on abdominal computed tomographic scan in blunt trauma. [Medline].1998; 44(5):889-92.

3. Jan.Holmes JF, Offerman SR, Chang CH, Randel BE, Hahn DD, Frankovsky MJ, et al. Performance of helical computed tomography without oral contrast for the detection of gastrointestinal injuries. Annals Emerg Med. 2004; 43(1):120-8.

4. Christiano JG, Tummers M, Kennedy A. Clinical significance of isolated intraperitoneal fluid on computed tomography in pediatric blunt abdominal trauma. J Pediatric Surg. 2009; 44(6):1242-8.

5. Shanmuganathan K. Multi-detector row CT imaging of blunt abdominal trauma. Seminar Ultrasound CT MR. 2004; 25(2):180-204.

6. Wing V, Federle M, Morris J. The clinical impact of CT for blunt abdominal trauma. Am J Roentgenol. 1985; 145:1191-1194.

7. Udean J Abdominal Trauma, Blunt (Serial online) Available from: http://www.emedicine.com/med/ topic2804.htm. Accessed: 27 Feb 2003 .
8. Robert A, Novelline M, James T, et al. Helical CT in Emergency Radiology. Radiology. 1999; 213:321-339.

9. Sultan Qaboos Univ Med J. 2007 Apr; 7(1): 41-46.

10. Federle M, Jeffery R. Hemoperitoneum studied by computed tomography. Radiology. 1983; 148:187192.

11. Norman L, John B. The symptoms, signs and emergency management of major injuries; Browse's :4th Ed 2005 2;43.

12. Kevin McConnell \& Douglas J.E Schuerer. Trauma Surgery : The Washington Manual of Surgery.5th Ed . 2008; 22: 373 .

13. Peitzman A, Makaroun M, Slasky B, et al. Prospective study of computed tomography in initial management of blunt abdominal trauma. J Trauma. 1986;26:585-592. [PubMed].

14. Webster V. Abdominal trauma: Pre-operative assessment and postoperative problems in intensive care. Anaesthesia Intensive Care. 1985;13:258262.

15. Lang E. Intra-abdominal and retroperitoneal organ injuries diagnosed on dynamic computed tomograms obtained for assessment of renal trauma. J Trauma. 1990;30:1161-1168.

16. Matsubara $\mathrm{T}$, Fong $\mathrm{H}$, Burns C. Computed tomography of abdomen (CTA) in management of blunt abdominal trauma. J Trauma. 1990; 30:410414.

17. Taylor GA, Fallat ME, Potter BM, et al. The role of computed tomography in blunt abdominal trauma in children. J Trauma. 1988;28:1660-1664. [PubMed].

18. Nolan B, Gabram S, Schwartz R, et al. Mesenteric injury from blunt abdominal trauma. Am Surg. 1995;61:501-506.

19. Kane N, Dorfman G, Cronan J. Efficacy of CT following peritoneal lavage in abdominal trauma. $\mathrm{J}$ Comp Assist Tomogr. 1987;11:998-1002. 